\title{
INHIBITORY EFFECT OF RED PIPER BETEL LEAF ETHANOL EXTRACT (PIPER CROCATUM RUIZ AND PAV.) AGAINST TRICHOMONAS VAGINALIS TROPHOZOITES IN VITRO
}

\author{
SRI AGUNG FITRI KUSUMA ${ }^{1 *}$, WIDYASTUTI S MANAN ${ }^{2}$, FAJAR BUDIMAN ${ }^{1}$ \\ ${ }^{1}$ Department of Pharmaceutical Biology, Faculty of Pharmacy, Padjadjaran University, Sumedang 45363, West Java, Indonesia. \\ ${ }^{2}$ Department of Parasitology, Medical Faculty, University of Indonesia, Jakarta, Indonesia. Email: s.a.f.kusuma@unpad.ac.id
}

Received: 16 June 2017, Revised and Accepted: 04 August 2017

\section{ABSTRACT}

Objective: The objective of this study is to determine the minimum inhibitory concentration (MIC) of red betel leaf ethanol extract against Trichomonas vaginalis trophozoites.

Materials and Methods: The samples of red piper betel leaf were extracted with $70 \%$ ethanol using a maceration method. The phytochemical screening of ethanol extract was performed using the standard procedures. Isolation of T. vaginalis trophozoites from vaginal secretions was conducted using swab method, then suspending it into a saline solution sterile and followed by observing trophozoites motility using a microscope. While the anti-leucorrhea activity test and determination of MIC extract was done using macrodillution method. The inhibitory effect of each extract concentration to trichomonas trophozoites' morphology was observed under a microscope using staining techniques by adding Giemsa dyes.

Results: The extract of red piper betel leaves contains several secondary metabolites such as alkaloids, flavonoids, polyphenols, quinones, and saponins. Of the six vaginal secretions, four positive trichomoniasis secretions were obtained. The results of anti-leucorrhea activity of red piper betel ethanol extract showed that the number of inactive trophozoites was proportional to the increasing of extract concentration. The inactive trophozoites were characterized by the absence of its motility under microscope observation. The MIC value of red piper betel extract of T. vaginalis trophozoites was $5 \% \mathrm{w} / \mathrm{v}$.

Conclusion: It can be concluded that the ethanol extract of red piper betel leaves could be contribute as a natural and safe anti-leucorrhea candidate for in vivo study on trichomoniasis in the future.

Keywords: Leucorrhea, Trichomonas vaginalis, Trophozoites, Red piper betel.

(C) 2017 The Authors. Published by Innovare Academic Sciences Pvt Ltd. This is an open access article under the CC BY license (http://creativecommons. org/licenses/by/4. 0/) DOI: http://dx.doi.org/10.22159/ajpcr.2017.v10i11.20778

\section{INTRODUCTION}

Trichomonas vaginalis is one of the most common sexually transmitted pathogens in the world, with an estimated 170 million cases occurring each year [1]. It is generally whitish, yellowish, or greenish vaginal discharge in females that might be normal or a symptom of infection. It is almost mucus discharge, which exhibit exfoliation of vaginal epithelial cells due to estrogen influence on the vaginal mucosa. It is important to identify the differences between physiologic and pathologic discharges [2]. Symptoms include vaginitis and infections have been associated with preterm delivery, low birth weight, and increased infant mortality, as well as predisposing to HIV/AIDS and cervical cancer [3]. Trichomoniasis has the highest prevalence and incidence of any sexually transmitted infection. In 2005, a study about the prevalence and sexual risk behavior among female sex workers in nine provinces, Indonesia, reported that the prevalence of $T$. vaginalis was $15.1 \%$. Inconsistent condom use was common (73.2\%); starting sex work at a younger age, and higher number of clients were independent risk factors [4]. The age-specific prevalence of the disease was seen to differ from other sexually transmitted diseases in a number of studies. Parameter fitting indicates that the average duration of infection in women is at least 3-5 years and approximately 4 months for men. The transmission probability of this infection can be spread from male to female or from female to male [5]. Another study showed that between $85 \%$ and $100 \%$ of the female partners of a series of 120 men identified with $T$. vaginalis in two hospital clinics were also positive for T. vaginalis [6,7]. Studies have shown the number of infected male partners of infected women ranges from $4 \%$ to $80 \%$ [8].

Syndromic management, that is, treating only those with symptoms of the disease had minimal effect on the endemic prevalence of disease even at high levels of coverage. While screening for the identification of individuals with both symptomatic and asymptomatic infections, was shown to be the most efficient method of control, but was sensitive to the screening interval [5]. Awareness of the sexual workers to go to a clinical laboratory can reduce the spread of trichomoniasis disease. However, they are generally ashamed to go to a doctor and get checked out. Some of them do self-medication, so the treatment of trichomoniasis infection is not complete. This can trigger the occurrence of T. vaginalis resistance against antibiotics that have been effective.

Metronidazole and tinidazole are the recommended drugs to treat trichomoniasis. However, clinical isolates derived from treatment failures typically show resistance to metronidazole [3]. Besides that, metronidazole and tinidazole have their own side effects. One of the important side effects of metronidazole is its carcinogenic effect, but due to inadequate evidence, it is not regarded as a risk factor for cancer in man $[9,10]$. Hence, it is necessary to search for the effective alternative drugs with fewer side effects. One of the plants that have long been used to treat the vaginal discharge is a red piper betel leaf (Piper crocatum). In addition, the red betel leaf ethanol extract has been proven safe for the normal vaginal flora of Lactobacillus acidophillus and Lactobacillus bifidus [11].

\section{MATERIALS AND METHODS}

Materials

Fresh red piper betel leaves ( $P$. crocatum Ruiz and Pav.) were collected from Bogor, Indonesia. The determination of test plant was conducted in Plant Taxonomy Laboratory of Biology Major, Faculty of Mathematics and Natural Science, Padjadjaran University. 
Trophozoites of T. vaginalis were obtained from the vaginal secret of sexual workers in one of rehabilitation center in Indonesia. The components of culture medium were trypticase, maltose, bacto agar, L-cystein hydrochloride, horse blood, streptomycin, and penicillin. The chemicals that were used are ethanol $70 \%$, distilled water, amyl alcohol, ammonia, dimethyl sulfoxide (DMSO), chloroform, acetic acid solution, physiological $\mathrm{NaCl}$ solution $0.9 \%$, barium chloride solution, sulfuric acid solution, Lugol's solution, n-butanol (Bratachem), ferric chloride reagent, Dragendorff's reagents, Lieberman-Burchard reagent, Mayer and Giemsa dyes.

\section{Extraction}

The leaves of red piper betel were washed in tap water, chopped, and dried. The dried leaves were grounded manually in the grinder and powdered leaves were used for extract preparation. The extraction was done using the maceration method. The plant material $(1 \mathrm{~kg})$ was macerated with $21 \mathrm{~L}$ of $70 \%$ ethanol during $3 \times 24 \mathrm{hrs}$ with the replacement of solvent every $24 \mathrm{hrs}$. The extracts were evaporated using a rotary evaporator at $40-50^{\circ} \mathrm{C}$, then continued to evaporate on a water bath until dried extract with constant weight was obtained. The extract was stored in a refrigerator at $4{ }^{\circ} \mathrm{C}$ until time of use [12].

\section{Phytochemical screening}

The phytochemical screening was performed for alkaloids, flavonoids, quinones, polyphenols, tannins, saponins, steroids, monoterpenoid, triterpenoid, and sesquiterpenoid. The screening was done using Fansworth method [13]. The color intensity or the precipitate formation was used as analytical responses to these tests.

\section{Medium preparation}

The medium used consisted of trypticase, maltose, bacto agar, L-cystein hydrochloride, methylene blue, and distilled water. These materials except methylene blue were placed in Erlenmeyer. Then, put it on fire and left to boil. After boiling, filtered with filter paper and added distilled water. To this mixture, added methylene blue, and then its $\mathrm{pH}$ was measured using $\mathrm{pH}$ paper up to $\mathrm{pH}$ 6.00. Then, the medium solution was divided into several tubes and sterilized using an autoclave.

\section{Horse serum isolation}

The horse serum was required as one of media component. A total of $300 \mathrm{~mL}$ horse blood was accommodated in several heparin tubes. The coagulation of blood plasma was done using centrifugation at $3000 \mathrm{rpm}, 4^{\circ} \mathrm{C}$ for 2 minutes. The position of serum was on the top of the centrifugation result, so it could be separated easily from the red blood cell pellets using micropipette. The obtained serum was stored at $-4^{\circ} \mathrm{C}$ (freezer temperature).

\section{Isolation of trophozoites}

The trophozoites of T. vaginalis were isolated from the vaginal secretions of sexual workers in one of the rehabilitation centers in Indonesia. Screening for the presence of trophozoites was performed on six sexual workers. Vaginal secretion of each patient was taken by opening the vaginal lips using a vaginal speculum, and then the secret was taken by swabbing it using cotton buds sterile. The cotton buds were then dipped in a sterile physiological $\mathrm{NaCl}$ solution. The suspension secretions were then applied to the glass object and examined under a microscope to determine the presence of $T$. vaginalis by observing the shape and motility of trophozoites. The active trophozoites were selected for further inoculation.

\section{Trophozoites inoculation}

Before the medium was used, it was boiled first so that the methylene blue can be remixed and then allowed it to cool. The horse serum and Pen Strep antibiotics (penicillin and streptomycin) were added into the media. The addition of this antibiotic was aimed to inhibit the growth of bacteria that may exist also in vaginal secretions. Then, the secrets containing active trophozoites were inoculated into the growth of broth medium and incubated at $37^{\circ} \mathrm{C}$ for $18-24 \mathrm{hrs}$. The number of trophozoites in each suspension was calculated using the observation of the count chamber. The secrets sample with the largest number of trophozoites was selected for anti-leucorrhea activity test.

\section{Anti-leucorrhea activity test}

The activity of anti-leucorrhea was carried out using a liquid medium. Extracts were diluted with DMSO transferred to the culture tubes to obtain final concentrations of 20,40,60, and $80 \% \mathrm{w} / \mathrm{v}$. Metronidazole $(80 \% \mathrm{w} / \mathrm{v})$ was used as a comparative drug. A volume of $5 \mu \mathrm{L}$ trophozoites suspension was added into test tubes. The media without trophozoites and extract was used for the negative control while the positive control was consisted of media with trophozoites inoculation. The DMSO as extract solvent was also tested against trophozoites. All media were then incubated at $37^{\circ} \mathrm{C}$ for $18-24 \mathrm{hrs}$. Anti-leucorrhea activity was shown by observing the motility of trichomonas trophozoites under a microscope. The observations were made in 2 days. The effect of each extract concentration to trophozoites cell shape can be observed using Giemsa staining. The result of anti-leucorrhea activity of red piper betel leaves extracts against trophozoites of T. vaginalis then was statistically analyzed using one-way ANOVA method.

\section{Minimum inhibitory concentration (MIC) determination}

Determination of MIC was performed to determine the minimum dose of red piper betel leaf ethanol extract which can still provide antileucorrhea activity against trophozoites of T. vaginalis. Anti-leucorrhea activity test was conducted using various dose levels as follows: $0.675 \%, 1.25 \%, 2.5 \%, 5 \%$, and $10 \% \mathrm{w} / \mathrm{v}$. MIC determination was done using a macrodillution method for 2 days observations. After 24 and $48 \mathrm{hrs}$, viable $T$. vaginalis were identified and counted microscopically on the basis of its motility.

\section{RESULTS AND DISCUSSION}

\section{Yield of the extract}

From the rendemen calculation, the extraction of red piper betel leaves yields of $16.13 \%$. Value yield is related to the amount of secondary metabolites that successfully attracted when the extraction process by comparing the weight of the extract thick with heavy bulbs.

\section{Phytochemical screening results}

Based on the results of phytochemical screening, the ethanol extract of red piper betel leaves contained alkaloids, flavonoids, polyphenols, quinones, and saponins. The same phytochemical result was reported for ethanol extract of green piper betel leaves that contained alkaloids, flavonoids, polyphenols, and saponins [14]. These tests reveal the presence of various bioactive secondary metabolites which might be responsible for their medicinal attributes [15]. This is evidenced in another study which revealed that ethanol extracts of Arbutus unedo leaf showed to be effective (growth inhibition rate [GI]: 100\% against T. vaginalis trophozoites in vitro because of flavonol and phenol content [16].

\section{Trophozoites isolation result}

Of the six vaginal discharge samples, four positive trichomoniasis secretions were obtained. One trichomoniasis patient has been treated using metronidazole so that active trophozoites could not be found under the microscope. Of the four secretions, a secretion with a high titer of active trophozoites was selected for further study. The number of active trophozoites cells per $10 \mu \mathrm{l}$ suspensions can be shown in Table 1.

Based on the data in the table, it can be seen that the culture of sample No. 1 has a high number of active trophozoites. This data were supported by the information from the rehabilitation center that the patient was just netted and has not been treated. Thus, culture of patient No. 1 was used as a tested parasite in this study.

\section{Anti-leucorrhea activity test result}

The results of anti-leucorrhea activity of red piper betel ethanol extract showed that the number of inactive trophozoite was proportional to the increasing of extract concentration. Measurement of this activity 


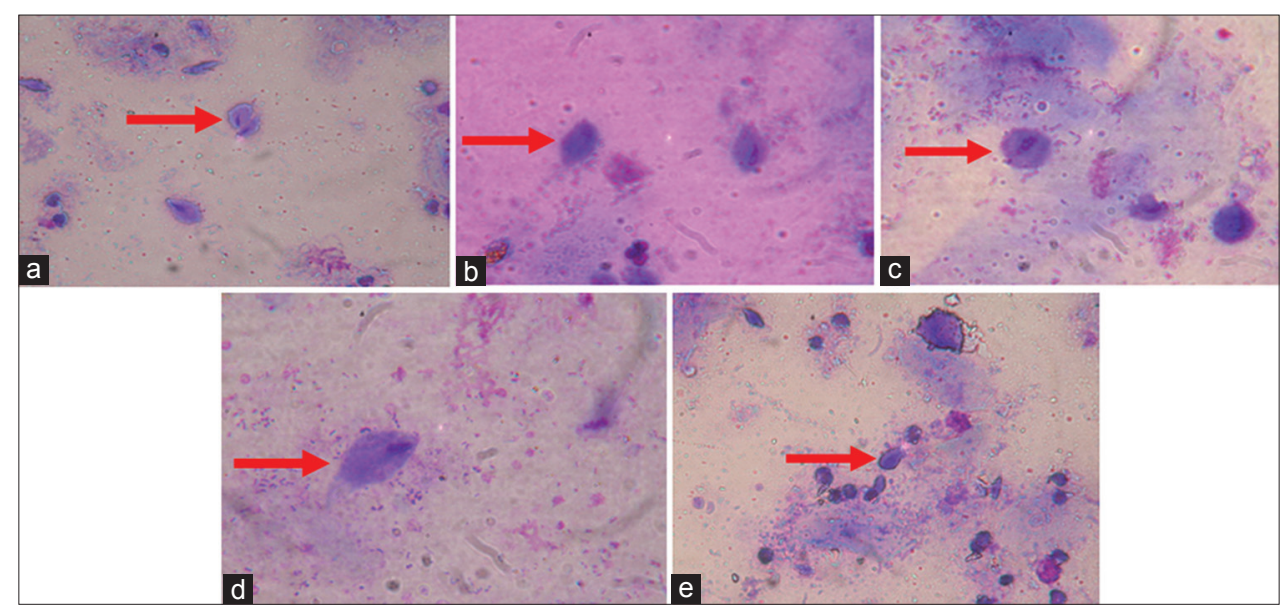

Fig. 1: The effect of extract in various concentrations on trophozoites shapes. (a) Without treatment; (b) $20 \% \mathrm{w} / \mathrm{v}$ extract; (c) $40 \% \mathrm{w} / \mathrm{v}$ extract; (d) $60 \% \mathrm{w} / \mathrm{v}$ extract; (e) $80 \% \mathrm{w} / \mathrm{v}$ extract

Table 1: The result of trophozoites counting in per $10 \mu \mathrm{l}$ suspensions

\begin{tabular}{lllll}
\hline Observation & \multicolumn{4}{l}{ Trophozoites number in each sample } \\
\cline { 2 - 5 } & $\mathbf{1}$ & $\mathbf{2}$ & $\mathbf{3}$ & $\mathbf{4}$ \\
\hline 1 & 15 & 6 & 6 & 9 \\
2 & 6 & 4 & 3 & 6 \\
3 & 7 & 3 & 2 & 7 \\
4 & 5 & 5 & 3 & 4 \\
5 & 6 & 3 & 4 & 3 \\
6 & 8 & 2 & 2 & 4 \\
7 & 4 & 4 & 3 & 3 \\
8 & 6 & 6 & 4 & 5 \\
9 & 7 & 6 & 5 & 4 \\
Total & 64 & 50 & 32 & 45 \\
\hline
\end{tabular}

Table 2: Anti-leucorrhea activity result

\begin{tabular}{lll}
\hline Treatment & Concentration $(\% \mathbf{w} / \mathbf{v})$ & $\begin{array}{l}\text { Active trophozoites } \\
\text { number }\end{array}$ \\
\hline Initial suspension & - & 32 \\
Extract & 80 & 4 \\
& 60 & 5 \\
& 40 & 7 \\
Metronidazole & 20 & 10 \\
Negative control & - & - \\
Positive control & - & - \\
DMSO control & & 46 \\
\hline
\end{tabular}

-: Active trophozoites absence, DMSO: Dimethyl sulfoxide

could not be done by comparing turbidity of test media, due to the determination of inactive trophozoites could only be observed under a microscope. The average number of active trophozoites within each test medium after 18 hrs incubation can be shown in Table 2 .

The result of the anti-leucorrhea activity of extract against trophozoites was analyzed using one-way ANOVA method could be shown in Table 3.

Based on the statistical analysis result, the $\mathrm{p}$ value corresponding to the F-statistic of one-way ANOVA is lower than 0.005, suggesting that the one or more treatments were significantly different. There was a significant decrease in the number of the trophozoites number of the initial suspension compared with trophozoites treatment with the extract in various concentrations. The ability of the extract is supported by the secondary metabolites contained in the extract. An increase in the trophozoites number in a positive control showed that the liquid
Table 3: Statistical analysis result

\begin{tabular}{llllll}
\hline Result & $\begin{array}{l}\text { Sums of } \\
\text { squares }\end{array}$ & df & Mean & $\begin{array}{l}\mathbf{F} \\
\text { statistic }\end{array}$ & p value \\
\hline Treatment & 63 & 3 & 6.5 & $<0.005$ & $0.000 \mathrm{e}+00$ \\
Error & 0 & 8 & 0 & & \\
Total & 63 & 11 & & & \\
\hline
\end{tabular}

Table 4: MIC result

\begin{tabular}{lll}
\hline Extract concentration $(\% \mathrm{w} / \mathrm{v})$ & $\begin{array}{l}\text { Active trophozoites } \\
\text { number in period of } \\
\text { incubation time (hrs) }\end{array}$ \\
\cline { 2 - 3 } & $\mathbf{2 4}$ & $\mathbf{4 8}$ \\
\hline 0.625 & 17 & 6 \\
1.25 & 13 & 4 \\
2.5 & 12 & 3 \\
5 & 10 & - \\
10 & 10 & - \\
\hline
\end{tabular}

-: Inactive, MIC: Minimum inhibitory concentration

medium used was the optimum media for trophozoites growth. It also could be proven that the DMSO did not gave inhibition effect on trophozoites of T. vaginalis. The inactive trophozoites as metronidazole's activity result showed that the clinical isolates of $T$. vaginalis used were still sensitive to metronidazole. The effect of extracts in various concentrations on trophozoites morphology could be seen clearly using Giemsa staining. There was no significant difference on trophozoites either active or inactive form because it only could be determined by observing its motility under a microscope. Trophozoites shapes after this treatment could be shown in Fig. 1.

\section{The result of MIC determination}

MIC value was determined as the smallest concentration of red piper betel leaves extract which able to inhibit the growth of T. vaginalis. By observing under a microscope, the smallest concentration of extracts that could still inhibit $T$. vaginalis was characterized by the absence of trophozoites motility. The observation result data can be shown in Table 4 .

The result showed that after $24 \mathrm{hrs}$ incubation of the parasite with the ethanol extracts in different concentrations, respectively, had higher GI. According to the results, the crude extract of the plant showed highest growth inhibitory effect on $T$. vaginalis after $48 \mathrm{hrs}$ incubation. It was found that the MIC value of red piper betel leaves extract was a range of $2.5-5 \% \mathrm{w} / \mathrm{v}$. It was reported in another study that red betel leaf extract at concentrations at range $2-4 \% \mathrm{w} / \mathrm{v}$ did not interfere to normal 
vaginal flora balance [11]. Therefore, the MIC value of this extract as anti-leucorrhea against trophozoites of T. vaginalis was safe and can be used as a reference dose for anti-leucorrhea preparation.

\section{CONCLUSION}

The results of this study showed that the ethanol extract of red piper betel leaves (P. crocatum) has anti-leucorrhea against trophozoites of T. vaginalis clinical isolates. The MIC of the extract was $5 \% \mathrm{w} / \mathrm{v}$. This herb can be introduced as new anti-leucorrhea herbal medicines for clinical research.

\section{REFERENCES}

1. World Health Organization. Global Prevalence and Incidence of Selected Curable Sexually Transmitted Diseases: Overview and Estimates. Geneva: World Health Organization; 1995

2. Dehdari S, Hajimehdipoor H. Herbal medicines for leucorrhea according to Iranian traditional medicine. Iran J Med Sci 2016;41 Suppl 3:S36

3. Dunne RL, Dunn LA, Upcroft P, O'Donoghue PJ, Upcroft JA. Drug resistance in the sexually transmitted protozoan Trichomonas vaginalis. Cell Res 2003;13(4):239-49.

4. Tanudyaya FK, Rahardjo E, Bollen LJ, Madjid N, Daili SF, Priohutomo $\mathrm{S}$, et al. Prevalence of sexually transmitted infections and sexual risk behavior among female sex workers in nine provinces in Indonesia, 2005. Southeast Asian J Trop Med Public Health 2010;41(2):463-73.

5. Bowden FJ, Garnett GP. Trichomonas vaginalis epidemiology: Parameterising and analysing a model of treatment interventions. Sex
Transm Infect 2000;76(4):248-56.

6. Catterall RD, Nicol CS. Is trichomonal infestation a veneral disease? $\mathrm{Br}$ Med J 1960;1(5180):1177-9.

7. Catterall RD. Diagnosis and treatment of trichomonal urethritis in men. Br Med J 1960;2(5192):113-5.

8. Lanceley F, Mcentegart MG. Trichomonas vaginalis in the male; the experimental infection of a few volunteers. Lancet 1953;1(6762):668-71.

9. Meites E. Trichomoniasis: The "neglected" sexually transmitted disease. Infect Dis Clin North Am 2013;27(4):755-64

10. Bendesky A, Menéndez D, Ostrosky-Wegman P. Is metronidazole carcinogenic? Mutat Res 2002:511(2):133-44.

11. Kusuma SA, Tjitraresmi A, Susanti G. Antibacterial effect of red piper betel leaf (Piper crocatum Ruiz \& Pav) ethanol extract to Lactobacillus acidophilus and L. bifidus growth inhibition. Asian J Pharm Clin Res 2017; Special issue May:65-68.

12. Kusuma SA, Zuhrotun A, Meidina FB. Antibacterial spectrum of ethanol extract of Indonesian red piper betel leaf (Piper crocatum Ruiz \& Pav) against Staphylococcus species. Int J Pharm Sci Res 2016;7(11):448-52

13. Fansworth NR. Biology and phytochemical screening of plants. J Pharm Sci 1966;55 Suppl 3:263-4

14. Sapna S, Anju D, Sanju N. Pharmacognostical and phytochemical studies of Piper betle Linn leaf. Int J Pharm Pharm Sci 2016;8(5):222-6.

15. Manjulika Y, Sanjukta C, Sharad KG, Geeta W. Preliminary phytochemical screening of six medicinal plants used in traditional medicine. Int J Pharm Pharm Sci 2014;6 Suppl 5:539-42.

16. Ertabaklar H, Kivçak B, Mert T, Ozensoy Töz S. In vitro activity of Arbutus unedo leaf extracts against Trichomonas vaginalis trophozoites. Turkiye Parazitol Derg 2009;33(4):263-5. 